\title{
A CATALOGUE OF HARBOURS IN THE PROVINCE OF ĜIRSU/LAGAŠ DOCUMENTED IN THE ADMINISTRATIVE TEXTS OF THE UR III PERIOD
}

\author{
SERGIO ALIVERNINI \\ Oriental Institute of the Czech Academy of Sciences \\ Pod Vodárenskou věží 1143/4 - 182 00, Praha 8, Czech Republic \\ e-mail: alivernini@orient.cas.cz
}

As is well known, boat trips on rivers and canals were an essential part of Mesopotamian economy at the end of the 3rd millennium BC; the Tigris and Euphrates, with their tributaries and canals, served as major highways in Mesopotamia. In the Ur III period, inland traffic by waterway was very extensive (more so than interregional water transport). This paper aims at highlighting ports/harbours of the province of Girsu/Lagaš recorded in the administrative texts from the end of the 3rd millennium, by cataloguing the name of the places where these harbours are located.

Key words: Ur III, harbours, Giirsu, Lagaš, ships, boats.

\section{Introduction ${ }^{1}$}

The province of Giirsu/Lagaš, along with the capitals Uruk and Ur, was located in the farthest south portion of Mesopotamia. It consisted of the three districts of Girsu, Kinunir-NIĜIN and Gu'abba. It extended $80 \mathrm{~km}$ north to south and $40 \mathrm{~km}$ east to west, covering a total area of more than $3000 \mathrm{~km}^{2}$ (Yoffee 2005: 57). The Girsu district was the seat of the homonymous city (Ĝirsu/Tello), from where almost all of the known cuneiform texts originate, plus other smaller centres such as Kisura, Kalamsaga and Kimadasala. It was located to the north, on the borders of the province of Umma,

${ }^{1}$ This study was written as part of the research funded by the Czech Science Foundation as the project GA ČR 18-01897S 'Economic Complexity in the Ancient Near East. Management of Resources and Taxation in the 3rd and 2nd Millennium BC.' Abbreviations used in the paper are found on the website of the Cuneiform Digital Library Initiative (URL consulted on 1st February 2019, http://cdli.ox.ac.uk/wiki/abbreviations_for_assyriology); add AS (Amar-Sin), IS (Ibbi-Sin), ŠS (Š́u-Sin), Š (Šulgi). The texts are transliterated using the following conventions: Obv. = Obverse, Rev. $=$ Reverse. The "/" points out the end of line, whilst "//" an indented line. 
whose nearest centre seems to have been Apišal, situated on the Tigris. In the district of Kinunir-NIĜIN, also known as 'the banks of the canal flowing towards NIĜIN' (Waetzoldt 1997), lay the major centres of Lagaš (al-Hiba), Kinunir, NIĜIN (Zurghul), as well as Kiesa and Urub. The southernmost district was Gu'abba ('the sea shore'), where we find the towns of Gu'abba, 'Old' Gu'abba, ${ }^{2}$ Asuna, Hurim, Gukara. The province of Ĝirsu/Lagaš was crossed by the Tigris which represented its border to the east. From the town of Girsu up to the sea, the province was crossed by a 'canal flowing towards NIĜIN' (Carroué 1986).

At the end of the 3rd millennium, the transportation of people and goods via watercourses is attested in thousands of documents and evidenced in all Ur III provinces. This paper focuses on the province of Ĝirsu/Lagaš and it is aimed at cataloguing all the harbours ${ }^{3}$ of the province recorded in the documentation. More precisely, the chronological limits are those of the Ur III Dynasty.

As already observed by Lafont (2010: 174-178), boats from the province of Ĝirsu/Lagaš reached centres both within the province and outside. Although Ĝirsu ship and boat texts are rich enough in terms of quantity, they often fail to contain the basic information needed for the reconstruction of routes, such as travel days or the waterways used. ${ }^{4}$ Despite these difficulties, as it is well known, the documentation reveals that connections by watercourse involving the province of Ĝirsu/Lagaš were very substantial. Not only can this be shown by reference to the quantity of texts, but also, in particular, by a number of texts that explicitly inform us that goods were loaded onto boats in many areas of this province. As will be shown in the following pages, although the documentation rarely references to harbours ('kar'), ${ }^{5}$ several zones of Ĝirsu/Lagaš province had harbours where ships were loaded and/or unloaded. ${ }^{6}$ Such harbours were not only recorded to be in the main centres, but also in granaries, fields, villages, temples and mills.

2 'Old' Gu'abba was probably located on the former coastline which had receded over the years due to the accumulation of river sediments (De Maaijer 1998: 63).

${ }^{3}$ In English, there are several words that define a place where boats can be loaded or unloaded: dock, harbour, port, quay or wharf, but we do not know how these structures appeared physically in Ur III times. Therefore, it is impossible to use the correct word to define them. In order to simplify things, this paper will use the word 'harbour' which, according to the Webster's dictionary, is 'a part of a body of water protected and deep enough to furnish anchorage' and seems to be a more general definition.

${ }^{4}$ This lack of information is even more evident if we compare it with the documentation coming from the province of Umma. For a reconstruction of the hydrology of the Umma province, see Steinkeller 2001.

${ }^{5}$ See, for example, MVN 5185 (ŠS 3/xi/25). To the best of my knowledge, there is no specific study on the Sumerian 'kar', although it is clear that the general meaning is a place which furnish anchorage: for example, Steinkeller (2001: 36, fn. 51) translate 'quay'; the electronic Pennsylvania Sumerian Dictionary translates 'harbor, quay' (http://psd.museum.upenn.edu/nepsd-frame. html, URL consulted on January 10th, 2019). The Akkadian word for 'kar' is 'kāru' and, in the 2nd and 1st millennia, it could also have different meanings. For an overview of the meanings of 'kāru', see the Chicago Assyrian Dictionary, K, pp. 231-239.

${ }^{6}$ The unloading operation in Ĝirsu/Lagaš province ('ba-al-la') are, oddly enough, recorded just twice in the documentation: Farmer's Instruction 7.06 (AS 1///-) and ITT $58239(-/ / /-)$. 


\section{Harbours in Ĝirsu/Lagaš Province}

In the Girsu/Lagaš province, several documents record the fact that boats were loaded in various places in the province, with goods being of different typologies. The basic idea of this paper is that we can reconstruct the network of harbours by collecting all those texts that mention boats carrying goods. These texts, apart from the quantity of the goods loaded, may also refer to the location where loading operations took place. From this, one can possibly assume that these sites also featured as harbours used for loading and unloading goods. However, the texts describing the movement of goods on boats do not often contain information on the places of departure or arrival, limiting themselves to recording the amount of $\operatorname{goods}^{7}$ and the names of the responsible officials. ${ }^{8}$ Some texts, on the other hand, contain only the starting place and the names of the officials who gave and received the goods, ${ }^{9}$ while others only record the place of arrival. ${ }^{10}$ Moreover, some texts record just a list of boats with their respective loading capacity and the place where these boats are stationed. ${ }^{11}$ Nevertheless, all these texts allow us to provide a list with the name of the places where harbours were located. It is worth stressing that if one looks at the texts more closely, it is not that simple to decide whether a given geographical name is identical with the place where the goods mentioned in the text were in fact loaded. Consider, for example, MVN $6190($ S 40/-/-):

Obv. 1-5: 180.0.0 še gur lugal / še a-ša $a_{3} \mathrm{Na}-b a-s a_{6} / \mathrm{ki}{ }^{\mathrm{d}}$ Utu-mu-ta / giri ${ }_{3} \mathrm{Lu}_{2^{-}}{ }^{\mathrm{d}} \mathrm{Na}-$ $\mathrm{du}_{3}$-a / dumu KA.KA

Rev. 1-5: $\mathrm{mu} \mathrm{Lu}{ }_{2}{ }^{\mathrm{d}} \mathrm{Na}-\mathrm{du}_{3}-\mathrm{a}-\mathrm{S}{ }_{3} /$ kišeb Ur- ${ }^{\mathrm{d}} \mathrm{Al}-\mathrm{la} \mathrm{ma}_{2}-\mathrm{lah}_{5} /$ blank line / ma 2 -a si-ga / $\mathrm{mu} \mathrm{us}_{2}-\mathrm{Sa} \mathrm{e}_{2}$ Puzur $_{4}{ }^{\text {iš }}{ }^{\mathrm{d}} \mathrm{Da}$-gan ba-du 3

Seal: Ur- ${ }^{\mathrm{d}} \mathrm{Al}-\mathrm{la} /$ dumu Ur-zikum-ma $/ \mathrm{ma}_{2}-\mathrm{lah}_{5} /{ }^{\mathrm{d}} \mathrm{Nin}-\hat{g} i \check{s}-z i-d a$

Obv. 1-5: 180 (gur) of barley (measured in) royal gur / barley of the field of Nabasa / from Utu-mu / via Lu-Nadua / son of KA.KA

Rev. 1-5: on behalf of Lu-Nadua / seal of Ur-Alla the sailor / blank line / loaded onto a boat / Year after the year: the temple of Puzriš-Dagan was built

Seal: Ur-Alla / son of Ur-zikum / the sailor / of Ninĝišzida

In this case, we cannot assume that the barley was loaded in the 'field of Nabasa' because we have no information where this barley was actually loaded. This is an administrative document which records the origin of the barley and its way of transport, but it is not meant to record its itinerary.

${ }^{7}$ In some texts, only the goods are recorded, without the indication of the quantity. See, for example, ITT 36128 (Š $28 / \mathrm{xi} /-)$, where 31 workers for 13 days towed a boat loaded with leather for the bala (obv. 1-2: 31 guruš $\mathrm{u}_{4} 13-\mathrm{še}_{3} / \mathrm{ma}_{2}$ kuš bala-še ${ }_{3}$ gid $_{2}$-da). On bala, see later.

${ }^{8}$ See, for example, MVN 7303 (Š 40/-/-).

${ }^{9}$ See, for example, MVN 7148 ( $\left.\breve{S} 40 / \mathrm{i} /-\right)$.

${ }^{10} \mathrm{See}$, for example, ITT 36128 (Š 28/xi/-).

${ }^{11}$ See, for example, BPOA 637 (unknown date). 
On the other hand, there are texts that clearly state where the goods are actually loaded. There are two expressions that indicate that something is loaded onto a boat: 'ma ${ }_{2}$-a si-ga', 'loaded onto a boat', and 'ma ${ }_{2}$-a gar-ra', 'placed onto a boat'. An example of the expression ' $\mathrm{ma}_{2}$-a si-ga' is provided in the text SAT 1303 (Š 46/iv/-):

Obv. 1-5: 310.0 .0 še gur lugal / $i_{3}-d u b_{5} E_{2}$-gibil 4 -le-ta / maa-a si-ga / kišeb Ur-ur dumu A-tu / iti šu-numun

Rev. 1-3: giri ${ }_{3} \mathrm{Ba}-z i$ / ugula $\mathrm{Iš}_{3}-\mathrm{am}_{3} / \mathrm{mu} \mathrm{us}_{2}-\mathrm{Sa} \mathrm{Ur}-\mathrm{bi}_{2}-\mathrm{lum}^{\mathrm{ki}} / /$ ba-hul

Seal: Ur-ur / dub-sar / dumu A-tu

Obv. 1-5: 310 (gur) of barley (measured in) royal gur / from the granary of E-gibile (field) / loaded onto a boat / seal of Ur-ur son of A-tu / month 4

Rev. 1-3: via Bazi / the supervisor is $\mathrm{Iš}_{3}-\mathrm{am}_{3} /$ Year after the year: Urbilum was destroyed

Seal: Ur-ur / scribe / son of Atu

The text clearly records that over ninety tons of barley were loaded from E-gibil field. It is thus clear that this field had a place to load goods onto boats.

An example of the expression ' $\mathrm{ma}_{2}$-a gar-ra' is provided by the text DAS 34 (AS 8/iv/-)

\section{Tablet}

Obv. 1-4: 33 guruš / $u_{4}$ 21-še ${ }_{3} /$ geš ma $\mathbf{m a}_{2}$-a ĝar-ra // $\mathrm{u}_{3} \mathrm{ma}_{2}$ gid $_{2}$-da / $\mathrm{E}_{2}$-gibil gile-ta $_{4}$

Rev. 1-4: $\hat{G i r}_{2}$-su ${ }^{\mathrm{ki}}-\breve{s e}_{3} /$ dumu dab ${ }_{5}$-ba-me // giri ${ }_{3} \mathrm{Lu}_{2}$-gu $\mathrm{gu}_{3}-\mathrm{de}_{2}$-a / iti šu-numun / mu en Eridu ${ }^{\text {ki }}$ ba-a-hun

\section{Envelope}

Obv. 1-5: 33 guruš / $\mathrm{u}_{4}$ 17-šse $/$ dumu dab ${ }_{5}$-ba-me / ĝeš mat-a ĝar-ra // $\mathrm{u}_{3} \mathrm{ma}_{2}$ gid $_{2}$ da // $\mathrm{E}_{2}$-gibil 4 -le-ta

Rev. 1-5: $\mathrm{Gir}_{2}-\mathrm{su}^{\mathrm{ki}}-\breve{s}_{3} / \mathrm{kišeb} \mathrm{Lu}_{2}-\mathrm{gu}_{3}-\mathrm{de}_{2}-\mathrm{a} /$ blank space / iti šu-numun / mu En Eridu $^{\mathrm{ki}}$ ba-a-hun

Seal: $\mathrm{Lu}_{2}-\mathrm{gu}_{3}-\mathrm{de}_{2}-\mathrm{a} / \mathrm{dub}-\mathrm{sar} / \mathrm{dumu} \mathrm{Ur}-[\ldots]$

\section{Tablet}

Obv. 1-4: 33 workers / for 21 days / placed wood onto a boat // and (they) towed the boat / from E-gibile

Rev. 1-4: to Girsu / (they) are dumu dab5-ba (workers) // via Lugudea / month 4 / Year: En of Eridu was installed

\section{Envelope}

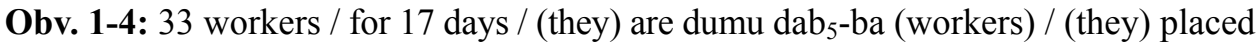
wood onto a boat // and (they) towed the boat // from the E-gibile 
Rev. 1-5: to Girsu / seal of Lugudea / blank space / month 4 / Year: En of Eridu was installed

Seal: Lugudea / scribe / son of Ur-[...]

In the latter case, 33 workers for 21 days (17 recorded on the envelope) placed wood onto a ship and towed it from E-gibile field to Girsu.

As the texts are not always clear regarding the place where goods were actually loaded, we must exercise cautions and distinguish among texts which record places where boats were loaded and others where we cannot prove this. More specifically, we can be sure that geographical name(s) mentioned in a text had a place we can define as 'harbour' in the following cases:

- texts which record that boats have been loaded in or from a specific place; ${ }^{12}$

- texts which record boats belonging to a specific place; ${ }^{13}$

- texts which record a number of workers for a number of days loading and/or towing a boat from a place to another. ${ }^{14}$

Keeping this in mind, the list below shows all places in the province of Girsu/ Lagaš that had a harbour, divided into settlement types and recorded in alphabetical order.

\section{Inhabited centres:}

\begin{tabular}{|c|c|c|c|c|}
\hline Harbour & Attestation & $\operatorname{Good}(\mathbf{s})^{15}$ & $\begin{array}{c}\text { Departure } \\
\text { place }\end{array}$ & $\begin{array}{c}\text { Arrival } \\
\text { place }\end{array}$ \\
\hline \multirow[t]{5}{*}{ Gir $_{2}$-su ${ }^{\text {ki } 16}$} & $\begin{array}{l}\text { CT } 5 \text { 39 BM } 17753 \\
\text { (AS 2/-/-) }\end{array}$ & $\begin{array}{l}288 \text { baskets and } \\
8 \text { tons of wool }\end{array}$ & $\mathrm{Gu}_{2}$-ab-ba ${ }^{\mathrm{ki}}$ & $\hat{\mathrm{G}} \mathrm{ir}_{2}$-su ${ }^{\mathrm{ki}}$ \\
\hline & $\begin{array}{l}\text { MVN } 12366 \\
\text { (AS 2/ix/-) }\end{array}$ & $\begin{array}{l}58.69 \text { tons of } \operatorname{sig}_{15} \\
\text { flour and } 492.3 \\
\text { tons of flour }\end{array}$ & $\hat{G} r_{2}$-su ${ }^{\mathrm{ki}}$ & Nibru $^{\text {ki }}$ \\
\hline & ITT 35102 (AS 7/vi/-) & & gišnimbar $\mathrm{du}_{3}-\mathrm{a}$ & $\hat{G i r}_{2}$-su ${ }^{\mathrm{ki}}$ \\
\hline & DAS 34 (AS 8/iv/-) & wood & $\mathrm{E}_{2}$-gibil ${ }_{4}-\mathrm{le}$ & $\hat{G i r}_{2}$-su ${ }^{\mathrm{ki}}$ \\
\hline & ITT 35084 (AS 9/v/-) & flour & $\mathrm{Gu}_{2}-\mathrm{ab}-\mathrm{ba}^{\mathrm{ki}}$ & 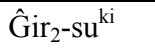 \\
\hline
\end{tabular}

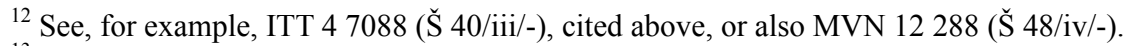

${ }_{13}^{13} \mathrm{See}$, for example, BPOA 637 (unknown date).

${ }^{14}$ See, for example, ITT 35102 (AS 7/vi/-). Sometimes, the place of arrival can be omitted: see, for example, MVN 5172 (ŠS 1/xi/-).

${ }^{15}$ The quantity of goods is converted into modern units of measurement. To simplify the conversion, the following equivalences are used: $1 \mathrm{gur}=300$ litres (ca. 300 kilograms), 1 ma-na = 500 grams.

${ }^{16}$ We have to highlight, however, that the name 'Ĝirsu' and the name 'Lagaš' can refer, in the Ur III texts, not only to the district or to the city but, in general, also to the province (Sallaberger and Westenholz 1999: 286). Therefore, in this case the texts might simply refer to the province, without specifying the district. 


\begin{tabular}{|c|c|c|c|c|}
\hline & ITT $35176($ AS 9/v/-) & flour & $\mathrm{Gu}_{2}-\mathrm{ab}-\mathrm{ba}^{\mathrm{ki}}$ & $\mathrm{Gir}_{2}-\mathrm{su}^{\mathrm{ki}}$ \\
\hline & $\begin{array}{l}\text { Fs. Owen 176 L. } 4976 \\
\text { (AS 9/xi/-) }\end{array}$ & semolina & $\hat{\mathrm{G}} \mathrm{ir}_{2}-\mathrm{su}^{\mathrm{ki}}$ & $\mathrm{Nibru}^{\mathrm{ki}}$ \\
\hline & UTI 42741 (ŠS 1/-/-) & & $\mathrm{Umma}^{\mathrm{ki}}$ & $\hat{G i r}_{2}$-su ${ }^{\mathrm{ki}}$ \\
\hline & $\begin{array}{l}\text { BPOA } 637 \text { (unknown } \\
\text { date) }\end{array}$ & & & \\
\hline & $\begin{array}{l}\text { ITT } 58239^{17} \text { (unknown } \\
\text { date) }\end{array}$ & barley & $\hat{\mathrm{Gir}}{ }_{2}-\mathrm{su}^{\mathrm{ki}}$ & $\hat{\mathrm{Gir}}{ }_{2}-\mathrm{Su}^{\mathrm{ki}}$ \\
\hline \multirow{6}{*}{$\mathrm{Gu}_{2}-\mathrm{ab}-\mathrm{ba}^{\mathrm{ki}}$} & ASJ 3183196 (Š 46/-/-) & $\begin{array}{l}8.839 \text { tons of se- } \\
\text { molina, } 104.999 \\
\text { tons of flour and } \\
1.02 \text { tons of } \operatorname{sig}_{15} \\
\text { flour }\end{array}$ & $\mathrm{Gu}_{2}$-ab-ba ${ }^{\mathrm{ki}}$ & \\
\hline & $\begin{array}{l}\text { CT } 5 \text { 39 BM } 17753 \\
\text { (AS 2/-/-) }\end{array}$ & $\begin{array}{l}288 \text { baskets and } 8 \\
\text { tons of wool }\end{array}$ & $\mathrm{Gu}_{2}-\mathrm{ab}-\mathrm{ba}^{\mathrm{ki}}$ & $\hat{\mathrm{Gir}}{ }_{2}-\mathrm{su}^{\mathrm{ki}}$ \\
\hline & TÉL 8 (AS 7/-/-) & flour & $\mathrm{Gu}_{2}-\mathrm{ab}-\mathrm{ba}^{\mathrm{ki}}$ & Nibru $^{\mathrm{ki}}$ \\
\hline & ITT 35084 (AS 9/v/-) & flour & $\mathrm{Gu}_{2}-\mathrm{ab}-\mathrm{ba}^{\mathrm{ki}}$ & $\hat{\mathrm{Gir}_{2}-\mathrm{su}^{\mathrm{ki}}}$ \\
\hline & ITT $35176($ AS 9/v/-) & flour & $\mathrm{Gu}_{2}$-ab-ba ${ }^{\mathrm{ki}}$ & $\hat{G}{ }_{2}$-su ${ }^{\mathrm{ki}}$ \\
\hline & $\begin{array}{l}\text { BPOA } 637 \text { (unknown } \\
\text { date) }\end{array}$ & & & \\
\hline $\mathrm{Ki}-\mathrm{es}_{3}-\mathrm{Sa}_{2}{ }^{\mathrm{ki}}$ & $\begin{array}{l}\text { BPOA } 637 \text { (unknown } \\
\text { date) }\end{array}$ & & & \\
\hline $\begin{array}{l}\text { Ki-ma-da-sal } 4^{-} \\
\mathrm{la}^{\mathrm{ki}}\end{array}$ & ITT 47072 (Š 30/x/-) & 30 litres of barley & & $\begin{array}{l}\text { Ki-ma-da- } \\
\text { sal }_{4}-\mathrm{la}^{\mathrm{ki}}\end{array}$ \\
\hline Ki-nu-nir ${ }^{\mathrm{ki}}$ & $\begin{array}{l}\text { BPOA } 637 \text { (unknown } \\
\text { date) }\end{array}$ & & & \\
\hline NIĜIN ${ }^{k i}$ & $\begin{array}{l}\text { BPOA } 637 \text { (unknown } \\
\text { date) }\end{array}$ & & & \\
\hline
\end{tabular}

Agricultural fields: ${ }^{18}$

\begin{tabular}{|c|l|l|l|c|}
\hline Harbour & \multicolumn{1}{|c|}{ Attestation } & \multicolumn{1}{|c|}{ Good(s) } & \multicolumn{1}{|c|}{$\begin{array}{c}\text { Departure } \\
\text { place }\end{array}$} & \multicolumn{1}{c|}{$\begin{array}{c}\text { Arrival } \\
\text { place }\end{array}$} \\
\hline a-ša ${ }_{3}$ a-ba-al-la* & $\begin{array}{l}\text { ITT } 47088 \\
(\check{S} 40 / \text { iii/- })\end{array}$ & $\begin{array}{l}59.76 \text { tons of } \\
\text { barley }\end{array}$ & a-ša ${ }_{3}$ a-ba-al-la & \\
\hline
\end{tabular}

${ }^{17}$ This text records a round trip from Girsu to Nippur: upstream (on the Tigris to Kasahar), turning at Kasahar, (then downstream) to Nippur, unloding the boat (in Nippur), upstream (to Kasahar, downstream on the Tigris) to Ĝirsu (Steinkeller 2001: 81).

${ }^{18}$ In the following lists, if the texts record that the goods were taken from the granary attached to a field or a village, the name of the field or village is marked with an asterisk ' $*$ '. 


\begin{tabular}{|c|c|c|c|c|}
\hline & $\begin{array}{l}\text { MVN } 7197 \\
\text { (Š 40/iii/-) }\end{array}$ & $\begin{array}{l}127.08 \text { tons of } \\
\text { barley }\end{array}$ & $\begin{array}{l}\left(i_{3}-\text { dub }_{5}\right) \text { a-ša } \\
\text { a-ba-al-la }\end{array}$ & \\
\hline & $\begin{array}{l}\text { Farmer's Instructions } \\
7.06 \text { (AS } 1 /-/-)\end{array}$ & $\begin{array}{l}3540 \text { tons of } \\
\text { barley }\end{array}$ & a-ša $a_{3}$ a-ba-al-la & $\mathrm{a}-\mathrm{ša}_{3}$ ha-zi \\
\hline a-ša ${ }_{3}$ a-KA-sahar & $\begin{array}{l}\text { MVN } 6483 \\
\text { (Š 40/vi/-) }\end{array}$ & $\begin{array}{l}72.36 \text { tons of } \\
\text { barley }\end{array}$ & a-ša $a_{3}$ a-KA-sahar & $\mathrm{Uri}_{5}{ }^{\mathrm{ki}}$ \\
\hline a-ša ${ }_{3}$ a-sag-du ${ }_{3}-d u$ & $\begin{array}{l}\text { MVN } 12288 \\
(\breve{S} 48 / \text { iv/-) }\end{array}$ & $\begin{array}{l}168.36 \text { tons of } \\
\text { barley }\end{array}$ & $\begin{array}{l}\left(i_{3}-d u b\right) a-s ̌ a_{3} a- \\
\text { sag-du }-d u\end{array}$ & \\
\hline $\mathrm{a}-\mathrm{ša}_{3} \operatorname{ambar}^{\mathrm{ki} *}$ & WMAH 9 (̌́ 47/v/-) & 51 tons of barley & $\left(i_{3}-d u b\right) A m b a r^{k i}$ & \\
\hline $\begin{array}{l}\text { a-ša }{ }_{3} \text { Ambar- } \\
\text { Lagaš }{ }^{k i}\end{array}$ & $\begin{array}{l}\text { MVN } 5172 \\
\text { (ك̌S 1/xi/-) }\end{array}$ & $\begin{array}{l}390 \text { litres of } \\
\text { barley }\end{array}$ & $\begin{array}{l}\left(\mathrm{a}-\check{s ̌ a}_{3}\right) \text { Ambar- } \\
\text { Lagaš }\end{array}$ & \\
\hline $\begin{array}{l}\text { a-ša }{ }_{3} \text { gišs̆ba-gibil- } \\
\text { la* }\end{array}$ & SAT 1287 (AS 2/-/-) & $\begin{array}{l}306.6 \text { tons of } \\
\text { barley }\end{array}$ & $\begin{array}{l}\mathrm{i}_{3} \text {-dub a-ša }{ }_{3}{ }^{\text {giš }} \text { ba- } \\
\text { gibil-la }\end{array}$ & \\
\hline a-ša ${ }_{3}$ bar-ra-AN* & $\begin{array}{l}\text { TCTI } 22692 \\
\text { (̌̌S 3/-/-) }\end{array}$ & $\begin{array}{l}9.645^{?} \text { tons of } \\
\text { barley }\end{array}$ & $\begin{array}{l}\left(i_{3}-d u b\right) \text { a-ša }{ }_{3} \text { bar- } \\
\text { ra-AN }\end{array}$ & \\
\hline a-ša $a_{3}$ BAR.AN* & $\begin{array}{l}\text { MVN } 1263 \\
(\check{S} 46 / i x /-) \\
\end{array}$ & $\begin{array}{l}87.9 \text { tons of } \\
\text { barley }\end{array}$ & $\begin{array}{l}\left(\mathrm{i}_{3}-\mathrm{dub}\right) \mathrm{a}- \\
\text { śa } \\
\mathrm{s}_{3} \text { BAR.AN }\end{array}$ & Nibru $^{k i}$ \\
\hline$a-\mathrm{sa}_{3}$ da-ze ${ }_{2}^{*}$ & $\begin{array}{l}\text { MVN } 12215 \\
(\check{S} 47 / \text { iii/-) }\end{array}$ & $\begin{array}{l}35.175 \text { tons of } \\
\text { barley }\end{array}$ & $\begin{array}{l}\left(i_{3}-d u b\right) a-s ̌ a_{3} \text { da- } \\
{z e_{2}}\end{array}$ & \\
\hline $\mathrm{a}$-ša ${ }_{3} d u_{6}$-imin & $\begin{array}{l}\text { MVN } 11114 \\
(\check{S} 47 /-/-)\end{array}$ & $\begin{array}{l}84.06 \text { tons of } \\
\text { barley }\end{array}$ & $a-\check{s ̆}_{3}{d u_{6}}_{6}-i m i n$ & \\
\hline \multirow{8}{*}{$\mathrm{a}-\mathrm{ša}_{3} \mathrm{e}_{2}-\mathrm{gibil}_{4}-\mathrm{le} *$} & 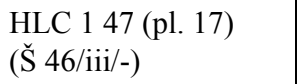 & 90 tons of barley & $\begin{array}{l}\left(\mathrm{i}_{3}-\mathrm{dub}\right) \mathrm{e}_{2}-\mathrm{gibil}_{4^{-}} \\
\mathrm{le}\end{array}$ & \\
\hline & $\begin{array}{l}\text { CM } 26 \text { no. } 83 \\
(\check{S} 46 / \mathrm{iii} /-)\end{array}$ & 90 tons of barley & $\begin{array}{l}\left(i_{3}-d u b\right) e_{2}-g i b i l_{4}- \\
\text { le }\end{array}$ & Nibru $^{k i}$ \\
\hline & $\begin{array}{l}\text { MVN } 1219 \\
(\breve{S} 46 / \text { iv/-) }\end{array}$ & $\begin{array}{l}281.4 \text { tons of } \\
\text { barley }\end{array}$ & $\begin{array}{l}\left(i_{3}-d u b\right) e_{2}-g_{i b i l}- \\
\text { le }\end{array}$ & Nibru $^{\text {ki }}$ \\
\hline & $\begin{array}{l}\text { MVN } 1220 \\
\text { (Š 46/iv/-) }\end{array}$ & $\begin{array}{l}149.4 \text { tons of } \\
\text { barley }\end{array}$ & $\begin{array}{l}\left(\mathrm{i}_{3}-\mathrm{dub}\right) \mathrm{e}_{2}-\mathrm{gibil}_{4^{-}} \\
\text {le }\end{array}$ & Nibru $^{\text {ki }}$ \\
\hline & SAT 1303 (Š 46/iv/-) & 93 tons of barley & $\left(i_{3}-\right.$ dub $) e_{2}-$ gibil $_{4}-\mathrm{le}$ & \\
\hline & $\begin{array}{l}\text { Nisaba } 1341 \\
(\check{S} 46 / \text { iv/-) }\end{array}$ & 270 tons of barley & $\begin{array}{l}\left(\mathrm{i}_{3}-\mathrm{dub}\right) \mathrm{e}_{2}-\mathrm{gibil}_{4^{-}} \\
\mathrm{le}\end{array}$ & \\
\hline & DAS 34 (AS 8/iv/-) & wood & $\mathrm{e}_{2}$-gibil 4 -le & $\hat{\mathrm{Gir}_{2}-\mathrm{su}^{\mathrm{ki}}}$ \\
\hline & $\begin{array}{l}\text { Fs. Owen 177 L. } \\
5182(\mathrm{AS} \mathrm{9/x/-)} \\
\end{array}$ & barley & $\mathrm{e}_{2}$-gibil 4 -le & Nibru $^{\text {ki }}$ \\
\hline a-ša $\operatorname{gan}_{2}-\mathrm{zi}^{*}$ & $\begin{array}{l}\text { TCTI } 22545 \\
\text { ( ̌SS 5/viii/-) }\end{array}$ & $\begin{array}{l}169.72 \text { tons of } \\
\text { barley }\end{array}$ & $\left(i_{3}-[d u] b\right) \operatorname{gan}_{2}-z i$ & \\
\hline \multirow[t]{3}{*}{ a-ša ${ }_{3}$ gi-dah-ha* } & $\begin{array}{l}\text { MVN } 1262 \\
\text { (Š 46/ix/-) }\end{array}$ & $\begin{array}{l}225.6 \text { tons of } \\
\text { barley }\end{array}$ & $\begin{array}{l}\left(i_{3}-d u b\right) a-s ̌ a_{3} \text { gi- } \\
\text { dah-ha }\end{array}$ & \\
\hline & $\begin{array}{l}\text { MVN } 1268 \\
\text { (Š 46/ix/-) }\end{array}$ & 219 tons of barley & $\begin{array}{l}\left.\text { (i } i_{3}-d u b\right) a-s ̌ a \\
\text { dah-ha }\end{array}$ & \\
\hline & ITT 36294 (IS 1/vi/-) & barley & a-ša $a_{3}$ gi-dah-ha & \\
\hline
\end{tabular}




\begin{tabular}{|c|c|c|c|c|}
\hline & LB 2366 (-/ix/-) & {$[\ldots]^{19}$ barley } & $\begin{array}{l}\left(i_{3}-d u b\right) a-s ̌ a_{3} \text { gi- } \\
\text { dah-ha }\end{array}$ & \\
\hline$a-s ̌ a_{3}$ ha-zi & $\begin{array}{l}\text { Farmer's Instructions } \\
7.06 \text { (AS } 1 /-/-)\end{array}$ & $\begin{array}{l}3540 \text { tons of } \\
\text { barley }\end{array}$ & $a-s ̌ a_{3}$ a-ba-al-la & $a-s ̌ a_{3}$ ha-zi \\
\hline $\begin{array}{l}\text { a-ša }{ }_{3} \text { in-im-bi-a- } \\
\text { ba* }\end{array}$ & $\begin{array}{l}\text { Nisaba } 1755 \\
(\check{\mathrm{S}} 45 / \mathrm{xi} /-)\end{array}$ & 171 tons of barley & $\begin{array}{l}\left(i_{3}-d u b\right) a-s ̌ a_{3} \text { in- } \\
\text { im-bi-a-ba }\end{array}$ & Nibru $^{\text {ki }}$ \\
\hline $\begin{array}{l}\mathrm{a}-\mathrm{ša} \\
\mathrm{LAGAB} \times \mathrm{GU} 4-\mathrm{a}\end{array}$ & $\begin{array}{l}\text { Nisaba } 17117 \\
\text { (Š 36/xii/-) }\end{array}$ & $\begin{array}{l}270.5 \text { litres of } \\
\text { barley }\end{array}$ & $\begin{array}{l}\text { a-ša } \\
\text { LAGAB } \times \text { GU4-a }\end{array}$ & \\
\hline $\mathrm{a}-\mathrm{ša}_{3} \mathrm{Sag}-\mathrm{ub}_{5}^{\mathrm{ki}}$ & $\begin{array}{l}\text { MVN } 7114 \\
(\breve{S} 40 / v i /-)\end{array}$ & 108 tons of barley & $a-\mathrm{s̆a}_{3} \mathrm{Sag}-\mathrm{ub}_{5}{ }^{\mathrm{ki}}$ & $\mathrm{Uri}_{5}{ }^{\mathrm{ki}}$ \\
\hline $\begin{array}{l}\text { a-ša }{ }_{3}{ }^{d} \breve{S} u l-g i-z i- \\
\text { kalam-ma }\end{array}$ & $\begin{array}{l}\text { MVN } 12398 \\
\text { (AS 4/i/-) }\end{array}$ & $\begin{array}{l}30.18 \text { tons of } \\
\text { barley }\end{array}$ & $\begin{array}{l}\text { a-ša }{ }_{3}{ }^{\mathrm{d}} \text { Šul-gi-zi- } \\
\text { kalam-ma }\end{array}$ & \\
\hline $\mathrm{a}-\mathrm{ša}_{3} \mathrm{SU}_{7}-\mathrm{MI}^{*}$ & $\begin{array}{l}\text { CM } 26 \text { no. } 92 \\
\text { (ŠS 3/-/-) }\end{array}$ & barley & $\begin{array}{l}\left(i_{3}-d u b\right) a-s ̌ a_{3} S U_{7^{-}} \\
\text {MI }\end{array}$ & $\mathrm{Nibru}^{\mathrm{ki}}$ \\
\hline
\end{tabular}

\section{Granaries: ${ }^{20}$}

\begin{tabular}{|c|c|c|c|c|}
\hline Harbour & Attestation & Good(s) & $\begin{array}{l}\text { Departure } \\
\text { place }\end{array}$ & $\begin{array}{c}\text { Arrival } \\
\text { place }\end{array}$ \\
\hline $\begin{array}{l}\mathrm{i}_{3} \text {-dub e } e_{2} \text {-gibil }{ }_{4}- \\
\text { le-tur }\end{array}$ & 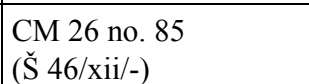 & $\begin{array}{l}200.7 \text { tons of } \\
\text { barley }\end{array}$ & $\begin{array}{l}\mathrm{i}_{3} \text {-dub } \mathrm{e}_{2} \text {-gibil } \mathrm{g}_{4} \text {-le- } \\
\text { tur }\end{array}$ & $\mathrm{Nibru}^{\mathrm{ki}}$ \\
\hline $\begin{array}{l}\mathrm{i}_{3}-\mathrm{dub} \mathrm{gu}_{2} \mathrm{i}_{7^{-}} \\
\text {niginin }\end{array}$ & SAT 1109 (AS 1/vii/-) & $\begin{array}{l}85.98 \text { tons of } \\
\text { hulled grain and } \\
900 \text { litres of barley }\end{array}$ & $\begin{array}{l}\mathrm{i}_{3} \text {-dub } \mathrm{gu}_{2} \mathrm{i}_{7^{-}} \\
\text {niĝin }\end{array}$ & \\
\hline $\begin{array}{l}\mathrm{i}_{3} \text {-dub KU-ki-ku- } \\
\text { ni } \hat{g}_{2}-\mathrm{du}_{10}{ }^{21}\end{array}$ & 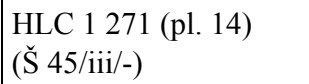 & 97.2 tons of barley & $\begin{array}{l}\mathrm{i}_{3} \text {-dub KU-ki-ku- } \\
\text { nig }_{2}-\mathrm{du}_{10}\end{array}$ & \\
\hline \multirow{2}{*}{$\begin{array}{l}\mathrm{i}_{3}-\mathrm{dub} \mathrm{KU}-\mathrm{ki}- \\
\text { nig }_{2}-\mathrm{du}_{10}\end{array}$} & $\begin{array}{l}\text { ASJ } 3166145 \\
(\check{S} 45 /-/-)\end{array}$ & 144 tons of barley & $\begin{array}{l}\mathrm{i}_{3}-\mathrm{dub} \mathrm{KU}-\mathrm{ki}- \\
\text { ni }_{2}-\mathrm{du}_{10}\end{array}$ & Nibru $^{\text {ki }}$ \\
\hline & $\begin{array}{l}\text { CM } 26 \text { no. } 82 \\
(\check{S} 45 /-/-) \\
\end{array}$ & 90 tons of barley & $\begin{array}{l}\mathrm{i}_{3} \text {-dub KU-ki- } \\
\text { nig }_{2}-\mathrm{du}_{10}\end{array}$ & Nibru $^{\text {ki }}$ \\
\hline \multirow[t]{3}{*}{$\mathrm{i}_{3}$-dub igi-gal ${ }_{2}$} & $\begin{array}{l}\text { OrSP } 54910 \text { Wengler } 5 \\
(\check{S} 46 / \mathrm{i} /-)\end{array}$ & $\begin{array}{l}112.92 \text { tons of } \\
\text { barley }\end{array}$ & $\mathrm{i}_{3}$-dub igi-gal ${ }_{2}$ & Nibru $^{k i}$ \\
\hline & $\begin{array}{l}\text { Nisaba } 1746 \\
\text { (ك̌ 46/xii/-) }\end{array}$ & 6.9 tons of barley & $\mathrm{i}_{3}$-dub igi-gal ${ }_{2}$ & Nibru $^{\text {ki }}$ \\
\hline & $\begin{array}{l}\text { MVN 12 } 194 \\
\text { (̌́ 47/i/-) }\end{array}$ & $\begin{array}{l}323.46 \text { tons of } \\
\text { barley }\end{array}$ & $\mathrm{i}_{3}$-dub igi-gal ${ }_{2}$ & Nibru $^{\text {ki }}$ \\
\hline
\end{tabular}

${ }^{19}$ The quantity is broken.

${ }^{20}$ This list records the granaries the names of which are not attributable to field names.

${ }^{21}$ This warehouse is attested just in this text. There might be a mistake in writing its name: KU-ki-ku-ni $\hat{g}_{2}-\mathrm{du}_{10}$ instead of KU-ki-nig $\hat{2}_{2}-\mathrm{du}_{10}$ (see the following row). 


\begin{tabular}{|c|c|c|c|c|}
\hline & MVN 12195 (Š 47/i/-) & $\begin{array}{l}271.38 \text { tons of } \\
\text { barley }\end{array}$ & $\mathrm{i}_{3}$-dub igi-gal ${ }_{2}$ & $\mathrm{Nibru}^{\mathrm{ki}}$ \\
\hline $\mathrm{i}_{3}$-dub $\mathrm{pa}_{5}$-enku & $\begin{array}{l}\text { Nisaba } 1346 \\
(\text { Š 46/ix/-) }\end{array}$ & 93 tons of barley & $\mathrm{i}_{3}$-dub $\mathrm{pa}_{5}$-enku & $\mathrm{Nibru}^{\mathrm{ki}}$ \\
\hline
\end{tabular}

\section{Villages:}

\begin{tabular}{|c|c|c|c|c|}
\hline Harbour & Attestation & Good(s) & $\begin{array}{c}\text { Departure } \\
\text { place }\end{array}$ & $\begin{array}{c}\text { Arrival } \\
\text { place }\end{array}$ \\
\hline $\mathrm{e}_{2}-\mathrm{duru}_{5} \mathrm{Ka}_{5}{ }^{\mathrm{a} *}$ & $\mathrm{MVN} 1216(\check{\mathrm{S}} 46 / \mathrm{ii} /-)$ & 58.8 tons of barley & $\mathrm{e}_{2}-\mathrm{duru}_{5} \mathrm{Ka}_{5}{ }^{\mathrm{a} *}$ & $\mathrm{Nibru}^{\mathrm{ki}}$ \\
\hline
\end{tabular}

Temples:

\begin{tabular}{|l|l|l|l|c|}
\hline \multicolumn{1}{|c|}{ Harbour } & Attestation & \multicolumn{1}{|c|}{ Good(s) } & \multicolumn{1}{c|}{$\begin{array}{c}\text { Departure } \\
\text { place }\end{array}$} & $\begin{array}{c}\text { Arrival } \\
\text { place }\end{array}$ \\
\hline $\mathrm{e}_{2}{ }^{\mathrm{d}} \mathrm{En}-\mathrm{ki}$ & $\mathrm{ITT} 35113(\mathrm{~S} \mathrm{~S} 4 / \mathrm{xi} /-)$ & barley & $\mathrm{e}_{2}{ }^{\mathrm{d}} \mathrm{En}-\mathrm{ki}$ & \\
\hline
\end{tabular}

\section{Other administrative structures:}

\begin{tabular}{|c|l|l|l|l|}
\hline \multicolumn{1}{|c|}{ Harbour } & \multicolumn{1}{|c|}{ Attestation } & \multicolumn{1}{|c|}{ Good(s) } & \multicolumn{1}{|c|}{$\begin{array}{c}\text { Departure } \\
\text { place }\end{array}$} & $\begin{array}{c}\text { Arrival } \\
\text { place }\end{array}$ \\
\hline $\mathrm{e}_{2}$-kikken & $\begin{array}{l}\text { Nisaba 18 } 91 \\
\text { (AS 8/iii/-) }\end{array}$ & flour & $\mathrm{e}_{2}$-kikken & \\
\hline
\end{tabular}

\section{Other places:}

\begin{tabular}{|c|c|c|c|c|}
\hline Harbour & Attestation & $\operatorname{Good}(s)$ & $\begin{array}{l}\text { Departure } \\
\text { place }\end{array}$ & $\begin{array}{l}\text { Arrival } \\
\text { place }\end{array}$ \\
\hline Ba-ba-az ${ }^{k i}$ & RA 621218 (ŠS 1/-/-) & & Ba-ba-az ${ }^{\text {ki }}$ & $\mathrm{e}_{2}$-gal TUM $\mathrm{TU}^{\mathrm{ki}}$ \\
\hline Bara $_{2}$-si-ga ${ }^{\text {ki } 22}$ & $\begin{array}{l}\text { Farmer's Instructions } \\
7.06 \text { (AS } 1 /-/-)\end{array}$ & $\begin{array}{l}3600 \text { tons of } \\
\text { barley }\end{array}$ & a-ša $a_{3}$ A-ba-al-la & \\
\hline
\end{tabular}

${ }^{22}$ Barasiga, probably identical with Barasiga of Hurim, belonged to the district of Gu'abba (Molina and Steinkeller 2017: 242, fn. 11). 


\section{Conclusions}

As can be seen from the above tables, harbours were located in all three districts of the province in both major ${ }^{23}$ and minor ${ }^{24}$ inhabited centres. Most of the documentation is extremely accurate, precisely recording the name of the field $\left(a-\check{s} a_{3}\right)$ where the goods were loaded. ${ }^{25}$ Other harbours can be identified in administrative units such as granaries $\left(i_{3}-d u b\right)$, villages $\left(e_{2}-d u r u_{5}\right)$ and temples $\left(e_{2}\right)$. Therefore, we are in a position to assume that each administrative unit probably had a place for loading and unloading boats. We have ca. 35 harbours recorded in the administrative documentation of Ĝirsu/Lagaš province, with the majority of them placed in agricultural fields. As one could expect, the ships were mainly loaded with barley (or with products derived from barley such as flour). As for the quantity of goods, we should note that the load capacities of ships ${ }^{26}$ documented in the province are (as the chart below shows) 1.5 tons (5 gur), 1.8 tons (6 gur), 2.4 tons ( 8 gur $), 3$ tons (10 gur $), 6$ tons $(20$ gur $), 9$ tons ( 30 gur), 12 tons ( 40 gur), 15 tons (50 gur), 18 tons (60 gur), 27 tons (90 gur), 36 tons (120 gur), 54 tons (180 gur) and 108 tons (360 gur), but the most common one seems to be boats with a load capacity of 18 tons.

\begin{tabular}{|c|c|}
\hline Capacity & Texts \\
\hline 5 gur & Comptabilité 23 ([-/-/-]). \\
\hline $6 g u r$ & ASJ $2223(-/-/-)$ \\
\hline 8 gur & ASJ $2223(-/-/-)$. \\
\hline $10 \mathrm{gur}$ & $\begin{array}{l}\text { WMAH } 3 \text { (AS 1/-/-); CT } 1050 \text { BM 12248 (AS 7/xi/-); SAT } 1369 \\
\text { (AS 8/ii/-); CM 26 no. } 102 \text { (AS 8/iii/14); CM } 26 \text { no. } 97 \text { (AS 8/xii/19); } \\
\text { TCTI } 23355 \text { (ŠS 3/iv/17); AION } 3117607 \text { (ŠS 3/-/-); }\end{array}$ \\
\hline $20 \mathrm{gur}$ & 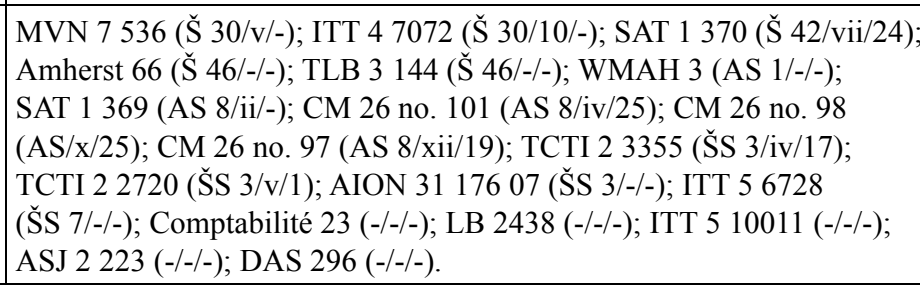 \\
\hline $30 \mathrm{gur}$ & 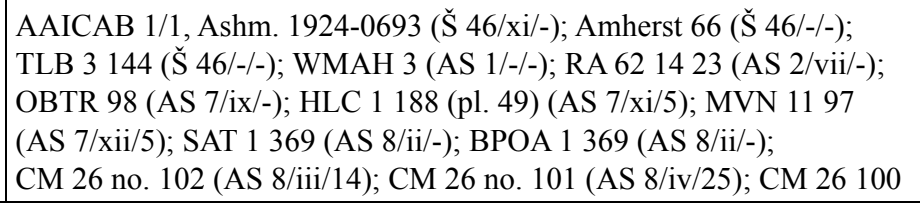 \\
\hline
\end{tabular}

${ }^{23}$ E.g. Ĝirsu or NIĜIN.

${ }^{24}$ E.g. Kiesa.

${ }^{25}$ It should be noted that texts often contain even more accurate information, indicating not only the name of the field but also, for example, that specific goods had been collected from the granary in the field itself $\left(\mathrm{i}_{3}-\mathrm{dub}_{5} \mathrm{a}^{\mathrm{a}} \mathrm{-s}_{3} x\right.$, 'granary in the field $x$ ').

${ }^{26}$ With regard to the capacity of boats, see Widdel 2009: 159-160. 


\begin{tabular}{|c|c|}
\hline & $\begin{array}{l}\text { (AS 8/iv/-); CM } 26 \text { no. } 99 \text { (AS 8/x/7); CM } 26 \text { no. } 98 \text { (AS 8/x/25); } \\
\text { TCTI } 1 \text { 922 (AS 8/xi/29); CM 26 no. } 97 \text { (AS 8/xii/19); TCTI } 11007 \\
\text { (AS 8/-/-); Comptabilité 52 (ŠS 2/vi/15); TÉL } 113 \text { (ŠS 2/-/-); WMAH } 4 \\
\text { (ŠS 4/x/-); ITT } 2892 \text { (ŠS 9/ii/-); PPAC } 51664 \text { (IS 2/-/-); PPAC } 5625 \\
\text { (-/vii/20); TCTI } 22785 \text { (-xii/12); TCTI } 22772 \text { (-/xii/17/); WMAH } 15 \\
\text { (-/8/-); HLC } 3384 \text { (pl. 145) ([-/-/-]); ASJ } 181679 \text { ([-/-/-]); RTC } 307 \\
\text { [(-/-/-]); BPOA } 637 \text { (-/-/-). }\end{array}$ \\
\hline $40 \mathrm{gur}$ & $\begin{array}{l}\text { TLB } 3144 \text { (Š 46/-/-); HLC } 1188 \text { (pl. 49) (AS 7/xi/5); MVN } 1197 \\
\text { (AS 7/xii/5); CT } 10 \text { 50 BM } 12248 \text { (AS 7/xi/-); SAT } 1369 \text { (AS 8/ii/-); } \\
\text { BPOA } 1169 \text { (AS 8/ii/-); CM } 26 \text { no. 102 (AS 8/ii/14); CM 26 100 } \\
\text { (AS 8/iv/-); CM 26 no. } 99 \text { (AS 8/x/7); CM 26 no. 98 (AS 8/x/25); } \\
\text { TCTI } 1922 \text { (AS 8/xi/29); TCTI } 11007 \text { (AS 8/-/-); BPOA } 1326 \text { (ŠS 5/-/-); } \\
\text { PPAC } 5625 \text { (-/vii/20); TCTI } 22785 \text { (-/xii/12); TCTI } 22772 \text { (-/xii/17/); } \\
\text { HLC } 3384 \text { (pl. 145) ([-/-/-]); Comptabilité 23 (-/-/-); ITT } 510011 \text { (-/-/-); } \\
\text { BPOA } 637 \text { (-/-/-). }\end{array}$ \\
\hline $50 \mathrm{gur}$ & $\begin{array}{l}\text { HLC } 1188 \text { (AS 7/xi/5); SAT } 1369 \text { (AS 8/ii/-); CM } 26 \text { no. } 102 \\
\text { (AS 8/iii/14); CM } 26100 \text { (AS 8/iv/-); PPAC } 5625 \text { (-/vii/20); HLC } 3384 \\
\text { (pl. 145) ([-/-/-]). }\end{array}$ \\
\hline $60 \mathrm{gur}$ & 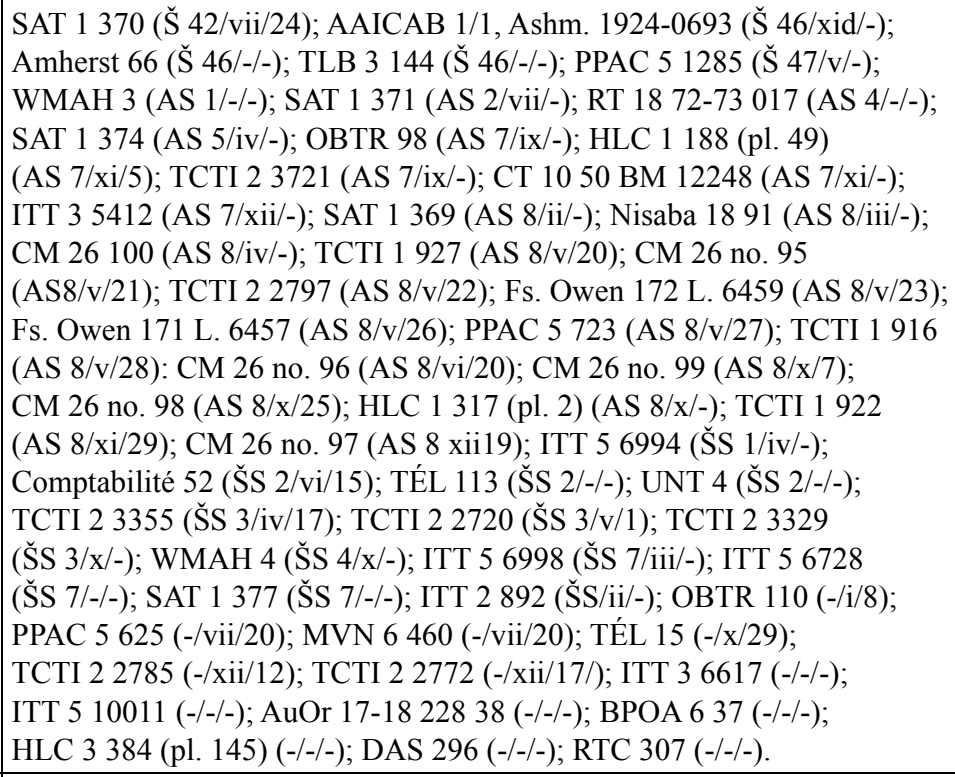 \\
\hline $90 \mathrm{gur}$ & HLC 3384 (pl. 145) (-/-/-); RTC 307 (-/-/-). \\
\hline $120 \mathrm{gur}$ & $\begin{array}{l}\text { CT } 1050 \text { BM } 12248 \text { (AS 7/xi/-); WMAH } 4 \text { (̌̌S 4/-/-); PPAC } 5625 \\
\text { (-/vii/20); TCTI } 22785 \text { (-/xi/12); ITT } 510011(-/-/-) \text {; BPOA } 637(-/-/-) ; \\
\text { HLC } 3384 \text { (pl. 145) (-/-/-). }\end{array}$ \\
\hline 180 gur & BPOA 637 (-/-/-); HLC 3384 (pl. 145) (-/-/-). \\
\hline 360 gur & BPOA 637 (-/-/-); HLC 3384 (pl. 145) (-/-/-). \\
\hline
\end{tabular}


As one can notice from the chart above, the amount of barley loaded is often considerably larger than the most common load capacity (and also larger than the maximum load capacity of 108 tons). This means that this massive amount of barley was probably loaded on more than one boat, although the texts do not record the number. Considering that, it is possible to state that every harbour had also a sort of 'fleet' where barley was loaded and sent to its final destination. Regarding this last point, as we can see from the chart, the two most documented destinations are Nippur and $\mathrm{Ur},{ }^{27}$ but these harbours were also important in linking centres within the province. ${ }^{28}$

\section{References}

CARrouÉ, François 1986. 'Le “Cours-d'Eau-Allant-à-NINA ${ }^{\mathrm{ki}, . '}$ Acta Sumerologica 8: 13-57.

LAFONT, Bertrand 2010. 'Sur quelques dossiers des archives de Girsu.' In: Alexandra KLEINERMAN and Jack SAsson (eds.) Why Should Someone Who Knows Something Conceal It? Cuneiform Studies in Honor of David I. Owen on His 70th Birthday. Bethesda: CDL Press, 167179.

De MaAiJer, Remco 1998. 'Land tenure in Ur III Lagaš.' In: Ben HARING and Remco De MAaiJer (eds.) Landless and Hungry? Access to Land in Early and Traditional Societies. Proceedings of a Seminar Held in Leiden 20 and 21 June 1996. Leiden: Research School CNWS, School of Asian, African, and Amerindian Studies, 50-73.

MolinA, Manuel and Piotr SteInKeller 2017. 'New Data on Garšana and the Border Zone between Umma and Girsu/Lagaš.' In: Llúis Feliu, Fumi Karahashi and Gonzalo RuBIO (eds.) The First Ninety Years. A Sumerian Celebration in Honor of Miguel Civil. [Studies on Ancient Near Eastern Record 12.] Boston and Berlin: De Gruyter, 231-249.

SAllaberger, Walther and Aage Westenholz 1999. Mesopotamien. Akkade-Zeit und Ur III-Zeit. [Orbis Biblicus et Orientalis 160/3.] Freiburg und Göttingen: Vandenhoeck \& Ruprecht.

Sharlach, Tonia 2004. Provincial Taxation and the Ur III State. [Cuneiform Monographs 26.] Leiden and Boston: Brill.

SteINKELLER, Piotr 2001. 'New Light on the Hydrology and Topography of Southern Babylonia in the Third Millennium.' Zeitschrift für Assyriologie und Vorderasiatische Archäologie 91: $22-84$.

YofFEe, Norman 2005. Myths of the Archaic State. Evolution of the Earliest Cities, States, and Civilizations. Cambridge: Cambridge University Press.

WAETzOLDT, Hartmut 1997. 'Die Bezeichnung gú-i -Nina $^{\mathrm{ki}}$-(šè)-du und die Verwaltungsbezirke der Provinz Lagaš.' Nouvelles Assyriologiques Brèves et Utilitaires 94/3: 87-89.

WidDel, Magnus 2009. 'Schiff und Boat.' In: Michael P. STRECK et al. (eds.) Reallexikon der Assyriologie und Vorderasiatischen Archäologie 12, Lieferung 1/2. Berlin und New York: de Gruyter, $158-160$.

${ }^{27}$ This is not surprising as the three provinces were linked by the 'bala' taxation system. For 'bala' and its connection with water transportation, see Sharlach 2004: 82-90. It is worth pointing out that most of the texts record shipments to the 'bala' to Nippur and only a few from Ur. Sharlach nevertheless observed that '[ $t$ ]he accounts of bala expenditures in Ur discussed above demonstrates large-scale expenditures in Ur, so again the paucity of documentation for shipments to Ur must be due to differential preservation of tablets' (Sharlach 2004: 86).

${ }^{28}$ See, for example, CT 539 BM 17753 (AS 2/-/-) which records a trip from Gu'abba to Ĝirsu, or DAS 34 (AS 8/iv/-) which records a trip from E-gibile field to Ĝirsu. 